\title{
Carbohydrate-induced stimulation of saccharin intake: Yoked controls
}

\author{
ISRAEL RAMIREZ \\ Monell Chemical Senses Center, Philadelphia, Pennsylvania
}

\begin{abstract}
Dilute, intragastric infusions of carbohydrate stimulate saccharin intake. To determine whether this effect is due to an associative process, a yoked control was employed. One group of rats was trained in the conventional fashion: they received carbohydrate (maltodextrin) infusions whenever they drank saccharin. Whenever these rats were infused with carbohydrate, yoked rats were infused with the same amount of carbohydrate. In an experiment in which saccharin was available continuously, carbohydrate infusions stimulated saccharin intake in conventionally trained rats but not in yoked rats. In a subsequent experiment, in which rats were offered saccharin for only $2.5 \mathrm{~h} /$ day, carbohydrate infusions stimulated intake in conventionally trained and in yoked rats. However, when these rats were switched to continuous saccharin availability, saccharin intake declined in yoked rats but remained elevated in conventionally trained rats. Thus, carbohydrate infusions stimulate saccharin intake via an associative process.
\end{abstract}

In studies designed to isolate the oral-sensory from the postingestive effects of carbohydrate solutions, it was found that rats given intragastric infusions of dilute carbohydrate solutions whenever they drank a saccharin solution drank considerably more than did rats infused with plain water (Ramirez, 1994b). The magnitude of the effect is impressive; rats infused with $3 \%-6 \%$ carbohydrate consumed 1.4-3 times as much saccharin as did rats infused with water (Ramirez, 1994b, 1996). Large effects also appeared when malto-oligosaccharide and $\mathrm{NaCl}$ were used instead of saccharin, but the effects were very much smaller and/or difficult to demonstrate when several other tastes/flavors were used, including cherry flavor, sucrose octaacetate, dilute maltodextrins, and monosodium glutamate (Ramirez, 1996). Although various forms of evidence suggest that carbohydrate-induced stimulation of intake is probably due to associative conditioning (Ramirez, 1996), such large differences between stimuli make one wonder whether some nonassociative effect is involved. Perhaps, for example, the presence of carbohydrate in the digestive tract induces an appetite for sweet. Support for this concept comes from the observation that carbohydrate infusions can, in an apparently nonassociative fashion, stimulate food intake (Geiselman, 1987; Geiselman \& Novin, 1982).

The importance of this point resides in its implications for the interpretation of the effect. A nonassociative effect would, by virtue of its inconsistency with what is known about appetite, seem like a laboratory artifact. Conversely, an associative mechanism would place the effect squarely within the literature on nutrient-conditioned taste preferences (E. W. Holman, 1975; G. L. Holman, 1969; Mehiel, 1991; Sclafani, 1990; Tordoff, 1991). Evidence that

Correspondence concerning this article should be addressed to I. Ramirez, Monell Chemical Senses Center, Philadelphia, PA 191043308. associative conditioning can greatly modify intake of sweet beverages would imply that learning is a more important determinant of ingestion than has previously been supposed.

If carbohydrate-induced stimulation of intake is an associative process, it should not occur when infusions are not contingent on the animal's ingestion of saccharin. In previous attempts to test this rats have been infused with carbohydrate on days when saccharin was not available (Ramirez, 1994b, 1996), but such experiments do not rule out the possibility that the nonassociative intakestimulating effects of carbohydrate infusions are shortlived and fade away by the time that saccharin has been made available. A more elegant way to test for nonassociative effects is to pair rats so that whenever a first rat ingests saccharin and is infused with carbohydrate, a second, yoked rat is infused. The yoked rat is free to ingest saccharin, but is only infused when its partner drinks. This technique cleanly removes the element of contingency, since infusions in yoked rats are unrelated to their behavior. Despite this absence of contingency, yoked rats receive the same infusions, at the same times, and at the same rates, as do the conventionally trained rats.

A potentially serious problem facing any such experiment arises from rats' ability to learn associations involving flavors even when there is a long delay between the response and the consequence. Since rats, in this laboratory, ingest mainly during the dark part of their light:dark cycle and show 10-60 bouts of drinking per day (Ramirez, 1997), yoked rats will often receive infusions within 15-30 min of drinking saccharin. Rats may be able to learn to associate a taste with a nutrient presented 30 or more minutes later (Capaldi, 1992; Capaldi, Campbell, Sheffer, \& Bradford, 1987; Elizalde \& Sclafani, 1988; E. W. Holman, 1975). Therefore, yoked rats might learn to increase saccharin intake by virtue of fortuitous pairings of saccharin ingestion and carbohydrate infusions. 
A factor that makes this problem even more troublesome is that rats can form flavor-nutrient associations even when the nutrient is presented before the flavor (Boakes \& Lubart, 1988). In the first experiment, massed trials (i.e., offering saccharin continuously) were employed in an attempt to minimize the effects of such fortuitous pairings - a procedure known to reduce the delay over which conditioning can take place (Gibbon, Baldock, Locurto, Gold, \& Terrace, 1977).

\section{EXPERIMENT 1}

\section{Method}

Animals. Twenty-four female $\mathrm{CD}$ rats $(\mathrm{Crl}: \mathrm{CD} \mathrm{BR}$, Charles River, Wilmington, MA) arrived in the laboratory when they were about 8 weeks of age. The rats were housed individually in hanging stainless steel cages, maintained on a 12:12-h light:dark cycle at $21^{\circ}-24^{\circ} \mathrm{C}$, and given Purina Laboratory Chow \#5001) ad lib. The rats were never deprived of food. When not given test fluids, the rats were given tap water in sipper bottles to drink ad lib. The rats always had some fluid to drink. The rats were maintained in the laboratory for at least $l$ week after arrival to permit them to acclimate to their surroundings before testing began.

Surgery. The catheters were like those described by Young and Deutsch (1981), except that they were made of 2-mm-diameter silicone rubber. They were implanted in a small hole placed in the forestomach, were sutured to both the stomach and the abdominal wall, exited the body through an incision in the back of the neck, and were anchored to the back of the neck with a stainless steel button. During surgery, the rats were anesthetized with ketamine $(90 \mathrm{mg} / \mathrm{kg})$ and acepromazine $(1 \mathrm{mg} / \mathrm{kg})$, supplemented with ether as needed. After surgery, the animals were treated with an analgesic (butorphanol $4 \mathrm{mg} / \mathrm{kg}$ ) and monitored daily for signs of infection or malaise. The rats were allowed to recover for at least 8 days after surgery before they were put in the test chambers.

Apparatus. The rats were housed in hanging stainless steel cages until they had recovered from surgery. Training was carried out in Plexiglas test chambers, each containing a Plexiglas rod with a $0.6-\mathrm{cm}$-diameter well that was filled with test fluid. Whenever a rat drank fluid from the well, a level detector activated a peristaltic pump that refilled the well and injected approximately the same amount of fluid into the rat's gastric catheter at $3.5 \mathrm{~g} / \mathrm{min}$ (Ramirez, 1996). A computer monitored the status of the pumps 18.2 times/ sec and stored the percentage of time in 10-sec bins.

Training. When the rats were placed in the test apparatus, their catheters were connected to an overhead tether with a swivel joint (Instech, Plymouth Meeting, PA). The rats were divided into three groups at this time. The control rats and the conventionally trained rats were infused with fluid every time they drank fluid; yoked rats were infused whenever the conventionally trained rats were infused. Fluid intake was measured daily.

The first 3 days were a habituation period in which all rats were offered plain water to drink and were infused with plain water. Beginning on the 4th day that the rats were in the apparatus, all rats were given $0.05 \%(2.4 \mathrm{mM})$ saccharin to drink. Control rats were infused with plain water whenever they drank saccharin. Conventionally trained and yoked rats were infused with $6 \%$ carbohydrate by weight (Maltrin M200, Grain Processing Corp., Muscatine, IA), which is similar to Polycose (Ramirez, 1994a). De-ionized water was used to make up solutions.

The rats were offered saccharin for a total of 7 days. Yoking was discontinued beginning on the 6th day of training; at this point, formerly yoked rats infused themselves in the same fashion as did the conventionally trained rats. On the 7 th day of training, all rats were infused with plain water (extinction test).
All procedures (changing solutions, flushing infusion lines, refilling food jars, etc.) were done in the light part of the light:dark cycle, about $4 \mathrm{~h}$ before the lights went out. The rats were permitted to drink and eat freely at all other times.

Statistics. All group data are given as means $( \pm S E)$. Intake of rats in the test apparatus is reported as grams ingested by mouth; conventionally trained rats received an equal amount of fluid via the gastric catheter. Computer records of intake were used to compute the amount of fluid ingested, and infused, over successive 30min periods.

Comparisons involving the control and other groups were made with a nonparametric analogue of the between-groups $t$ test; this method is variously known as permutation, randomization, or resampling (Edgington, 1987; Manly, 1991). Comparisons between the conventionally trained and yoked rats were done with the same nonparametric analogue of the paired $t$ test. All probability values are two-tailed.

\section{Results}

Total oral intake. During the initial infusion period, when all the rats were offered plain water to drink, there was a nonsignificant trend for yoked rats to ingest less water than did the other groups (all $p \mathrm{~s}>.06$; see Figure 1). When saccharin was offered and maltodextrin was being infused, conventionally trained rats ingested more than twice as much saccharin as did control rats on all days $(p \leq .05)$. Yoked rats never differed from control rats (all $p \mathrm{~s}>.08$ ) and consistently drank less than did the conventionally trained rats (all $p$ s $<.04$, except on the 3rd day of training when $p=.056$ ). Although intake did not change appreciably on the day of extinction (see Figure 1), probability values increased slightly, resulting in two-tailed $p$ values slightly greater than $5 \%$ (control vs. conventionally trained, $p=.07$; conventionally trained vs. yoked, $p=.06$ ).

Because of the trend for yoked rats to drink less than conventionally trained rats during the water-only period, data were recomputed as intake for each day minus intake on the last day before saccharin was offered. Comparisons between conventionally trained and yoked rats, on days when maltodextrin was infused, were all significant $(p<.05)$ except on the 3 rd day of maltodextrin infusion $(p=.06)$.

Patterns of ingestion within test days. To examine the degree to which intake by yoked rats was correlated with that of conventionally trained rats, intake for each pair of rats was plotted (Figures 2 and 3 show individual data from the 1 st and 5 th days of training). For most rats, drinking occurred in distinct bouts; this caused the individual intake curves to appear jagged. The size and number of bouts varied substantially among rats although there was a tendency for individual rats to show similar patterns across days (compare Figures 2 and 3 ). Because it is difficult to estimate total intake from such jagged curves, total oral intake for each rat is given in the upper left of each panel; group means on successive 30-min periods are shown in Figure 4. There was a trend for ingestion to be greater during the dark part of the light:dark cycle; this trend may be seen more easily when group means are plotted as in Figure 4. 

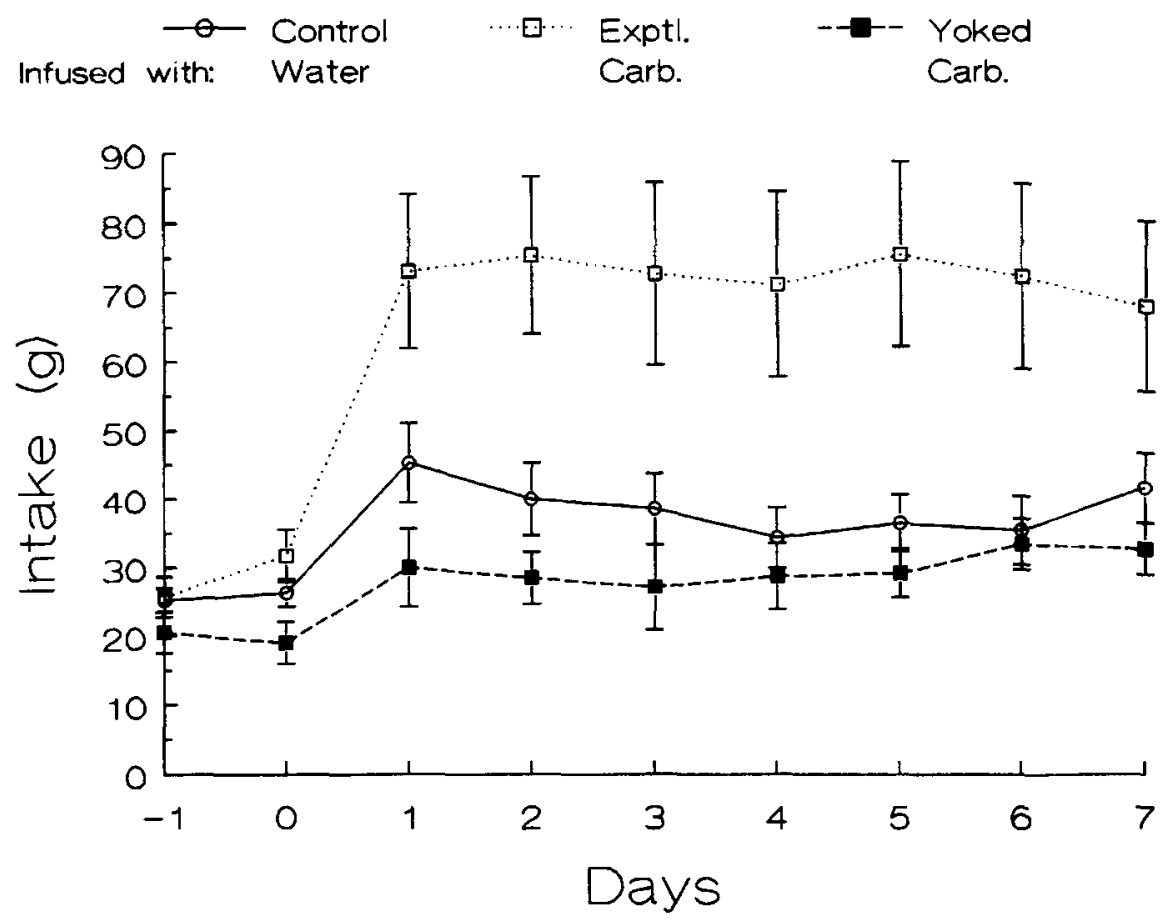

Figure 1. Mean $( \pm S E M)$ daily oral intake in the first infusion experiment. Throughout the period depicted, oral intake was accompanied by an intragastric infusion of the same volume. Days -1 and 0 are the 2 days immediately before the day on which the saccharin was first offered. Beginning on Day 1 , the rats were offered $0.05 \%$ saccharin and infused with either water or $6 \%$ carbohydrate (Maltrin M200). The yoking procedure was discontinued on Day 6. All rats were infused with plain water on Day 7 (extinction).

Visual inspection of intake patterns for each pair of conventionally trained and yoked rats (Figures 2 and 3 ) does not reveal strong relationships between amounts ingested by members of each pair. A more rigorous technique is to compute correlation coefficients for intake by conventionally trained and yoked rats. These were computed for each pair of rats using intake over successive 30-min blocks (i.e., using the data depicted in Figures 2 and 3 ). Correlations were computed for each pair and then averaged. Standard errors for these correlations were computed from the observed variation among rats rather than from the theoretical distribution of the correlation statistic. Significance tests were computed by permutation $t$ tests (Edgington, 1987; Manly, 1991). These correlations were positive but low. During the 1st day of training, the mean correlation coefficient was $.15 \pm .06(p=.02)$. During the second day of training, the mean correlation coefficient was $.10 \pm .07(p>.2)$. By the 5 th day of training, the mean correlation reached $.21 \pm .02$ ). Mean correlations were higher when block sizes longer than $30 \mathrm{~min}$ were used. For example, with 60-min blocks, intake by conventionally trained and yoked rats correlated $.22 \pm .08(p=.02)$ and $.36 \pm .06$ ( $p=.008)$ on the 1 st and 5th days of training, respectively. Correlations were lower when smaller block sizes were used.

\section{Discussion}

As in previous studies (Ramirez, 1994b, 1996), maltodextrin infusions promptly and substantially stimulated saccharin intake by conventionally trained rats. In contrast, yoked rats drank about as much saccharin as did control rats, and substantially less saccharin than did conventionally trained rats. One might propose that yoking reduces the effectiveness of some nonassociative, intakestimulating, effect of carbohydrate via various processes described by Church (1964). However, the data provide no support for such a view. If yoking merely attenuated the effectiveness of maltodextrin infusions, one would expect a gradual increase in intake over days in yoked rats. Instead, yoked rats drank $3 \%$ less saccharin on the last day of yoking (Day 5) than on the 1 st day of saccharin availability (Day 1 in Figure 1). Furthermore, when yoking was discontinued, group means did not greatly change-an observation that suggests learned helplessness.

$A$ basic assumption underlying this study is that the amounts and times at which yoked rats were infused were unrelated to the amounts and times at which they drank saccharin. Correlational analysis of intake in successive 30 -min periods indicates that this assumption is approximately correct. There are correlations between conventionally trained and yoked rats, but these are attributable to the tendency for all groups to drink more when room 

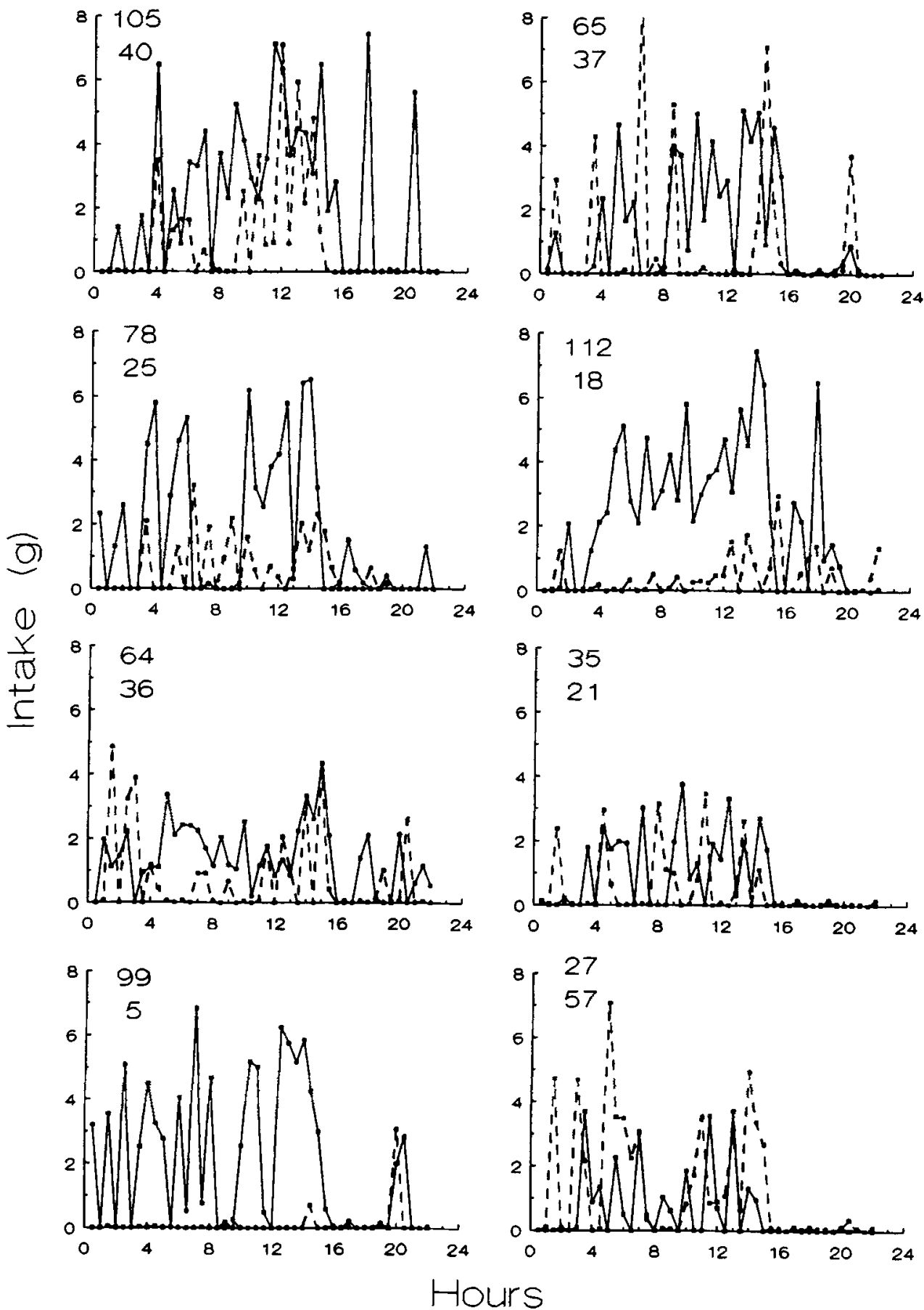

Figure 2. Oral intake over successive 30-min periods during the 1st day on which saccharin was available in Experiment 1 . Individual pairs of conventionally trained (solid lines) and yoked (dashed lines) rats are depicted in each panel. Total intakes for conventionally trained (upper) and yoked rats (lower) are shown in the upper left of each panel.

lights were off than when they were on. These correlations are extremely small, yielding coefficients of determination $\left(r^{2}\right)$ ranging from .01 to .05 . The magnitude of the correlation coefficient varied with the size of the block over which intake was computed. This is because positive correlations reflect diurnal patterns of ingestion; longer block sizes average out variation due to moment- to-moment changes in ingestion and thereby increase the apparent effect of diurnal variations in ingestion. The present report emphasizes 30 -min intervals because in most reports of successful flavor-nutrient conditioning, conditioned stimulus-unconditioned stimulus delays were 30 min or less (Capaldi, 1992; Capaldi et al., 1987; Elizalde \& Sclafani, 1988; E. W. Holman, 1975). 

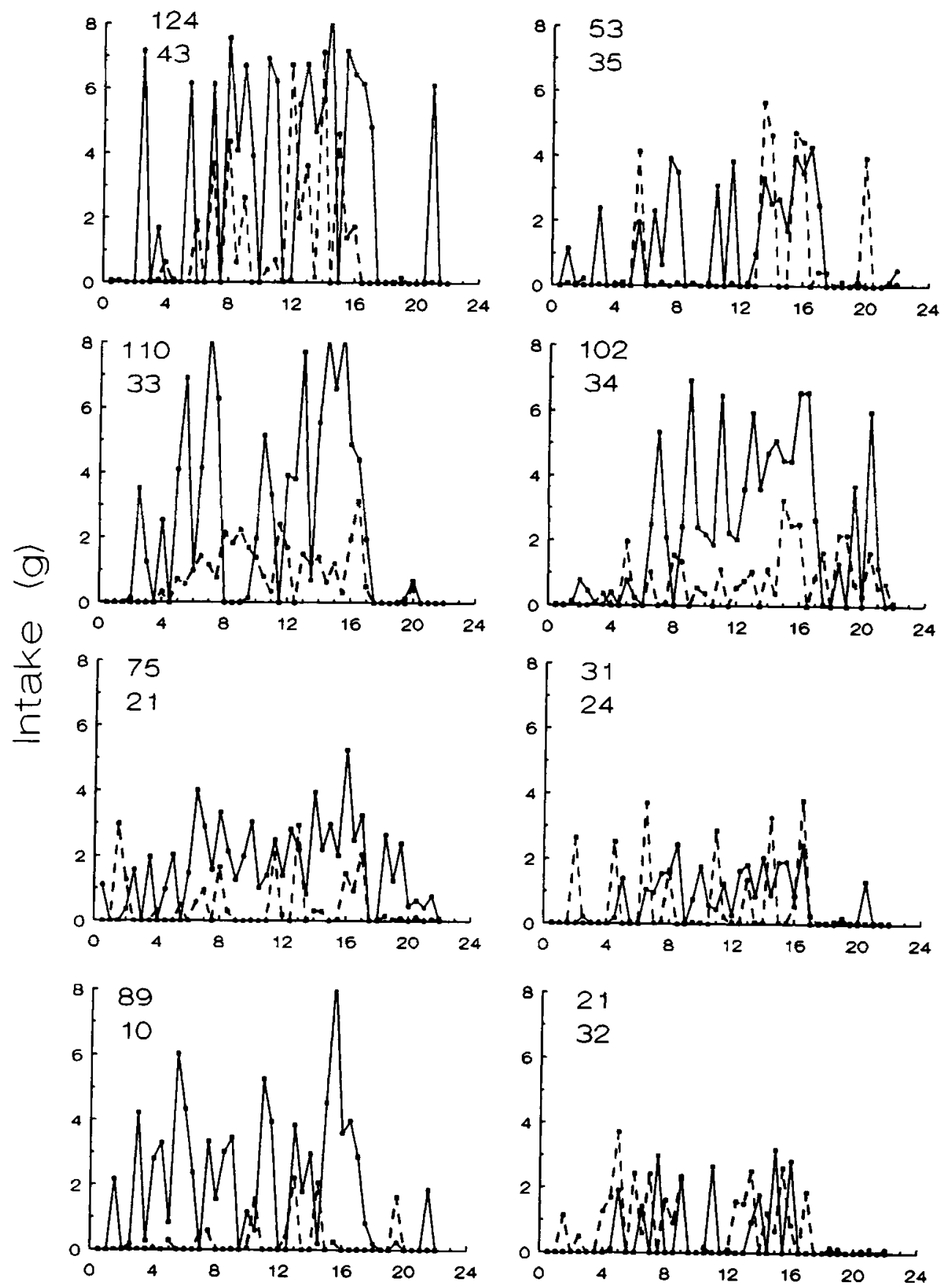

Hours

Figure 3. Oral intake over successive 30-min periods during the 5th day on which saccharin was available in Experiment 1. Individual pairs of conventionally trained (solid lines) and yoked (dashed lines) rats are depicted in each panel. Total intakes for conventionally trained (upper) and yoked rats (lower) are shown in the upper left of each panel.

\section{EXPERIMENT 2}

In studies of flavor-nutrient conditioning, it is common for training trials to be interspersed with exposure to a stimulus that is not reinforced. This was done in Experiment 2 by offering the rats saccharin for $2.5 \mathrm{~h}$ a day and plain water the rest of the day. Because this produced apparent conditioning in the yoked group, the rats were subsequently switched to massed trials (i.e. continuous saccharin exposure).

\section{Method}

Twenty-four new rats were procured, housed, fed, watered, operated on, permitted to recover, and installed in the apparatus accord- 

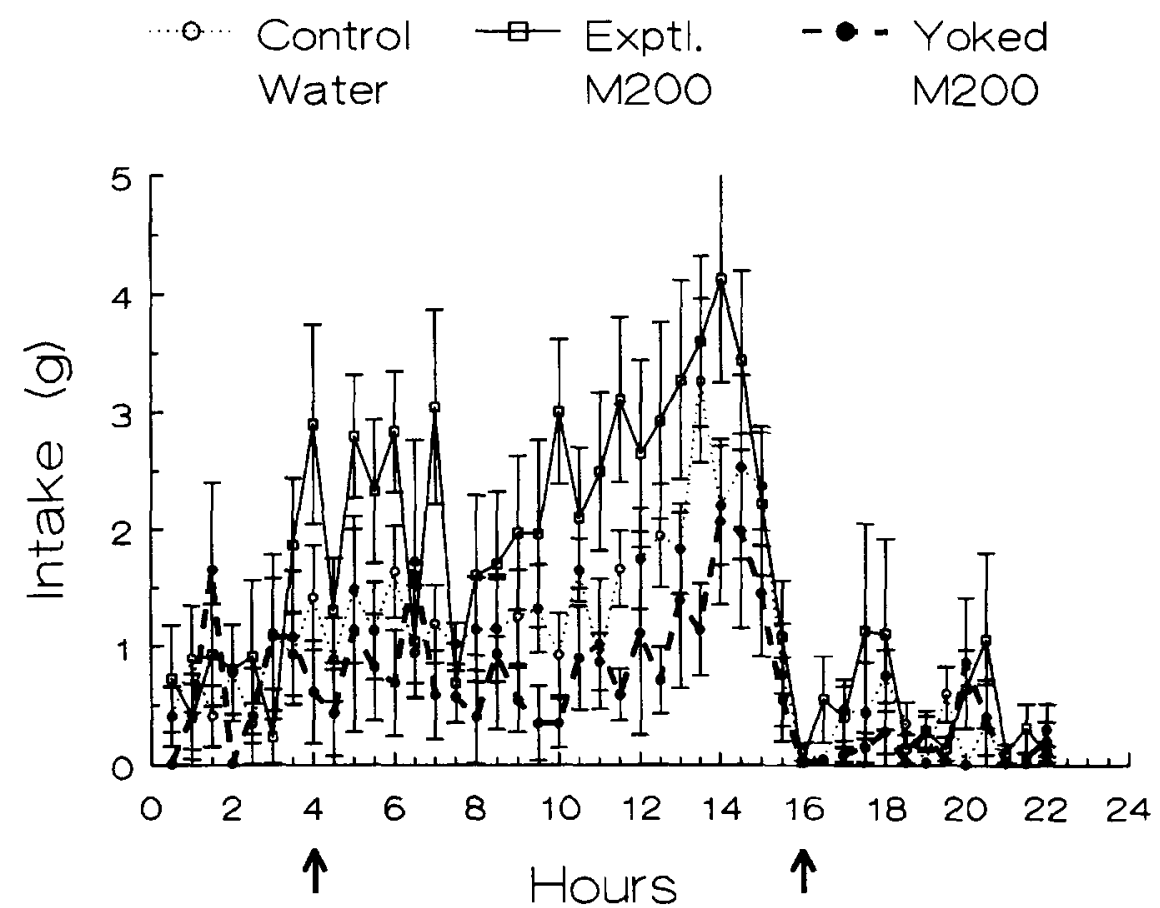

Figure 4. Oral intake $( \pm S E M)$ over successive 30 -min periods during the $1 \mathrm{st}$ day on which saccharin was available in Experiment 1. The arrows indicate when the lights went off (4 h) and on (16 h). Between 22 and $24 \mathrm{~h}$, the apparatus was serviced and no fluid was available.

ing to the same procedures as in Experiment 1. As in Experiment 1, the rats were divided into three groups: control, conventionally trained, and yoked. The rats were offered plain water and infused with plain water for the first 3 days.

Beginning on the 4 th day, the drinking wells were covered with an aluminum shroud $4 \mathrm{~h}$ before the dark part of the light:dark cycle began; the shrouds prevented the rats from drinking from the wells until the training-test period began. The shrouds were removed 1-5 min after the lights in the animal room went out, and the rats were permitted to drink for $2.5 \mathrm{~h}$. At the end of this period, the shrouds were replaced and the rats were given bowls with plain water; no fluids were infused when the rats drank from the bowls. The next day, the bowls were removed $4 \mathrm{~h}$ before the dark part of the light: dark cycle began, and the previous day's procedure was repeated. This limited access protocol was done for a total of 5 days. The first of these days was a practice day in which the rats were of fered plain water and were infused with plain water. On subsequent days, the rats were offered $0.05 \%$ saccharin. Conventionally trained and yoked rats were infused with $6 \%$ maltodextrin; control rats were infused with water. Beginning on the day after the fourth $2.5-\mathrm{h}$ session, the rats were offered saccharin continuously for 6 more days.

\section{Results}

The groups did not differ significantly $(p>.2)$ during the period when only plain water was offered, but there was a nonsignificant trend for yoked rats to drink more water than did the other groups (data not shown).

Conventionally trained rats did not differ from control rats when saccharin was first offered $(p>.2)$ but gradually increased saccharin intake on successive days, so that by the last 2.5 -h test, they drank significantly more saccharin than did control rats ( $p=.01$; see Figure 5).
Surprisingly, yoked rats drank more saccharin than did control rats $(p=.01, p=.08, p=.03, p=.002$, on the 1 st, $2 \mathrm{nd}$, 3rd, and 4th days, respectively). However, the difference between yoked and conventionally trained rats did not reach statistical significance (all $p \mathrm{~s} \geq$.1). In order to gain insight into the causes of the accelerated development of increased saccharin ingestion in yoked rats, intake records were examined by computer to identify the first bout of ingestion. A bout was defined as at least $0.1 \mathrm{~g}$ of intake separated from other bouts (before and after) by at least 5 min of no drinking. It was found that the very first bout of ingestion was greater in yoked than in conventionally trained rats $(4.1 \pm 0.8 \mathrm{vs} .1 .9 \pm 0.5 \mathrm{~g}$, respectively, $p=.04$ ). The first experiment also showed a trend in the same direction but it was not significant $(2.1 \pm 0.4$ vs. $1.4 \pm 0.5 \mathrm{~g}$, respectively, $p>.2$ ).

Rats drank at a high rate when saccharin was available (see Figure 6). As in Experiment 1, the correlation between intake of conventionally trained and yoked rats over successive 30 -min periods was computed for each pair. Such correlations were near zero when computed using data from the $2.5-\mathrm{h}$ period of saccharin availability only, but were substantial when computed for the entire period over which the rats had some fluid to drink; the latter method is the same as that used in Experiment 1. The mean correlation using the latter method of computation was $.52 \pm .08$ for the 1 st day of training, and $.74 \pm .05$ for the 2 nd day of training.

When saccharin was made available for the entire day, both conventionally trained and yoked rats drank more 


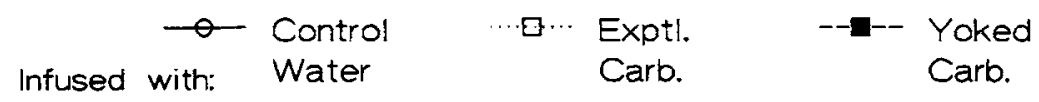

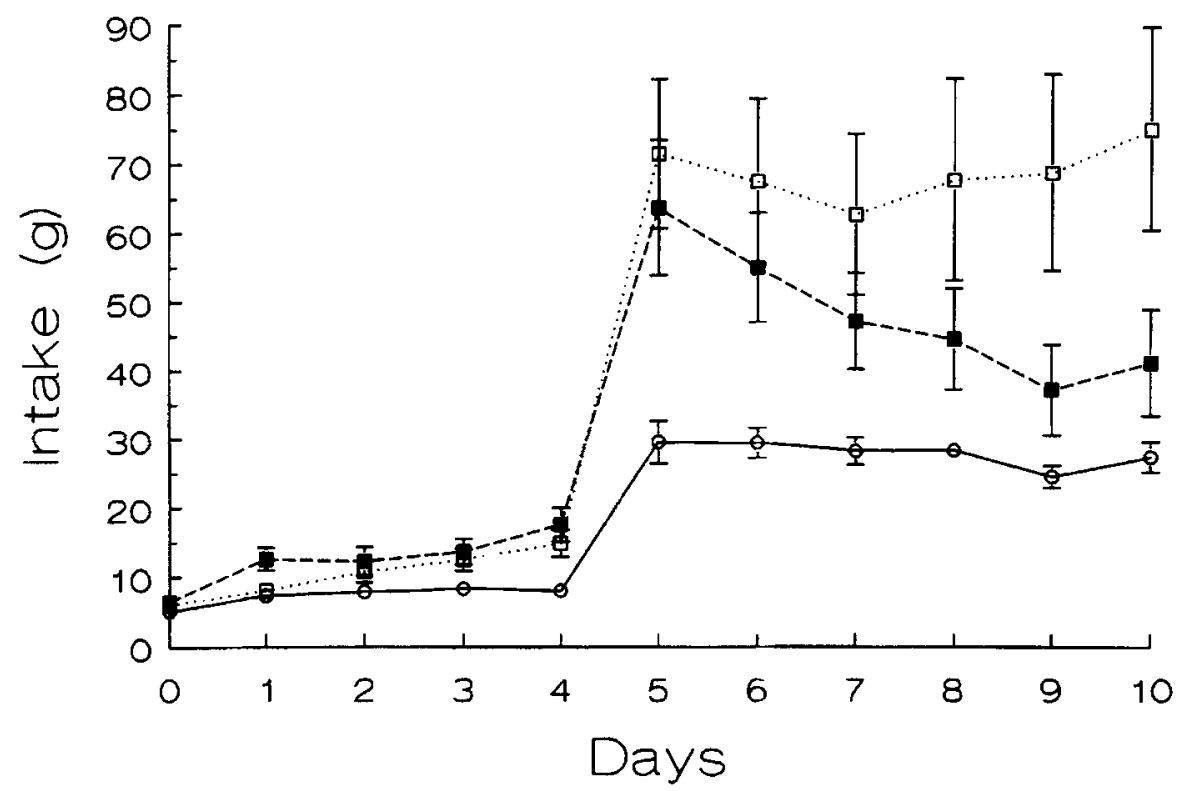

Figure 5. Mean ( $\pm S E M$ ) oral intake during the second infusion experiment. Throughout the period depicted, oral intake was accompanied by an intragastric infusion of the same volume. Rats were offered water to drink on Day 0 , and $0.05 \%$ saccharin on all other days. Saccharin was available for 2.5 h/day on Days 1-4, and for 23 h/day on Days 5-10. Days -1 and 0 are the 2 days immediately before the day on which the saccharin was first offered. Beginning on Day 1 , the rats were offered $0.05 \%$ saccharin and infused with either water or $6 \%$ carbohydrate (Maltrin M200). The yoking procedure was discontinued on Day 6. All rats were infused with plain water on Day 7 (extinction).

than twice as much saccharin as did control rats (see Figure 5). However, conventionally trained rats maintained a high level of intake over days, whereas yoked rats drank less saccharin over successive days (see Days 5-10, Figure 5). Thus, conventionally trained rats drank more than did control rats on all of these days (all $p s \leq .007$ ), whereas the difference between yoked and control rats started out significant ( $p \leq .05$, Days 5-9), but ended up nonsignificant $(p=.1$, Day 10$)$. Conventionally trained and yoked rats did not differ significantly on any one day (all $p s \geq .1$ ), but conventionally trained rats drank $3.6 \pm 6.6 \mathrm{~g}$ more at the end of this period than at the beginning, whereas yoked rats drank $22.6 \mathrm{~g}$ less at the end of the period than at the beginning (test on change scores, $p=.008$ ).

On the 1 st day that saccharin was made available for the entire day, the mean correlation between intake of conventionally trained and yoked rats over successive 30 min periods was low but well above zero $(.28 \pm .06, p=$ $.008)$. The reasons for this correlation are readily apparent in Figure 7; both conventionally trained and yoked rats drank at a high rate when saccharin was first made available, increased intake when the lights went out, and decreased intake when the lights went on. On the subsequent day, the correlation was lower but still significant $(.20 \pm .05, p=.02)$.

\section{Discussion}

In the very first bout of saccharin ingestion, yoked rats drank more saccharin than did conventionally trained rats. The first experiment showed a similar trend, but the effect was weaker and not significant. In other studies employing conventionally trained rats, carbohydrate infusions did not significantly stimulate ingestion during the first few hours of training (Ramirez, 1994b, 1996, 1997). It therefore seems likely that the elevated intake was due to yoking per se rather than to the carbohydrate infusions. Perhaps the intake suppressing effect of infusing fluid is reduced when the infusions do not occur at the same time as fluid is ingested. Such an effect should be particularly strong when a rat suddenly switches from drinking modest volumes of plain water to larger volumes of saccharin.

It is likely that the elevated intake seen in the yoked rats on subsequent days was, at least partly, due to the same mechanisms that induce elevated intake in conventionally trained rats. When saccharin was available for only $2.5 \mathrm{~h}$ a day, and correlations were computed for the entire period in which the rat could drink some fluid, there was a strong correlation between availability of saccharin and amount of carbohydrate infused in yoked rats, even though drinking and infusions did not occur at exactly the same times. Since taste-nutrient conditioning 

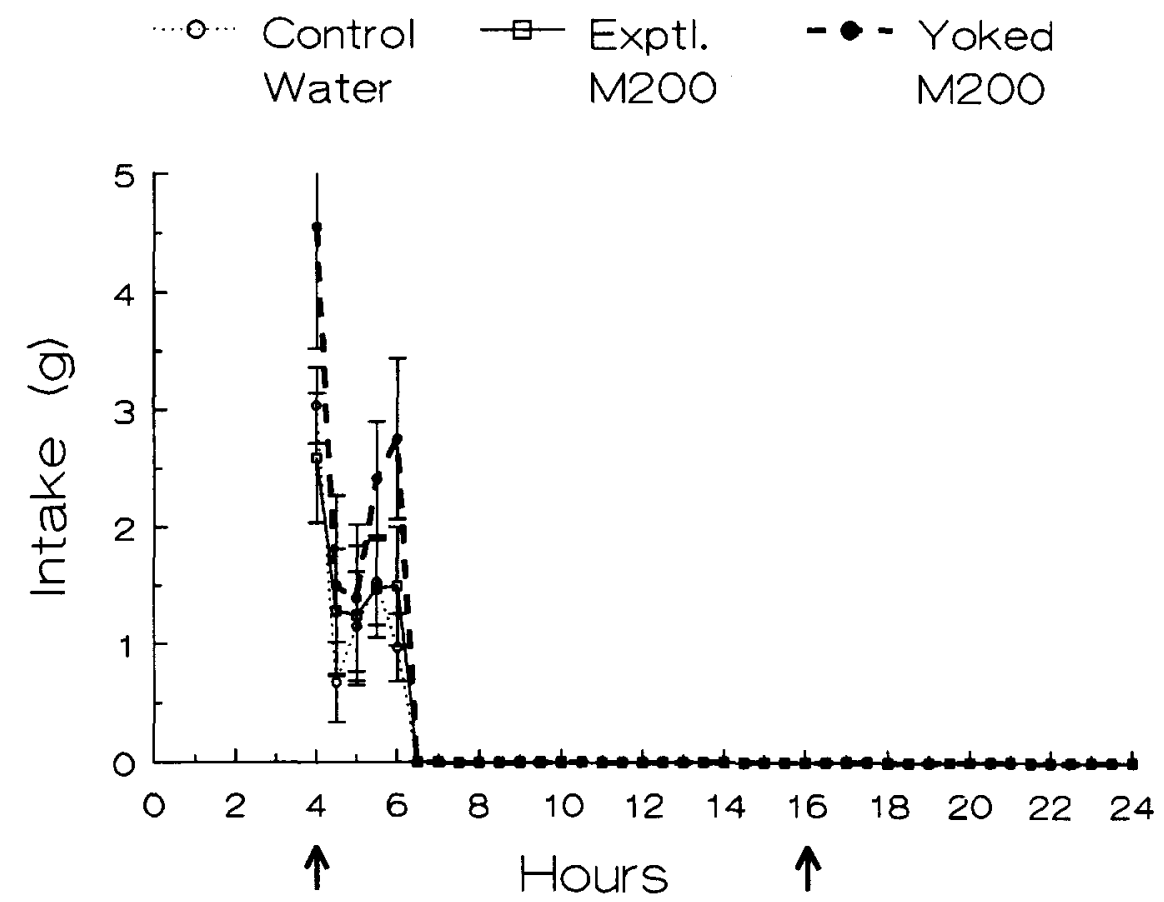

Figure 6. Oral intake $( \pm S E M)$ over successive 30-min periods during the 1 st day on which saccharin was available in Experiment 2. Saccharin was available between 4 and $6.5 \mathrm{~h}$. The arrows indicate when the lights went off $(4 \mathrm{~h})$ and on $(16 \mathrm{~h})$. No fluid was available for the first $4 \mathrm{~h}$ of the test session.

can occur over a relatively long conditioned stimulusunconditioned stimulus delay (Capaldi, 1992; Capaldi et al., 1987; Elizalde \& Sclafani, 1988; E. W. Holman, 1975), an ingestive response may be conditioned by merely making saccharin availability and carbohydrate infusions occur at approximately the same time of day. This interpretation is supported by the observation that when saccharin was offered all day long, saccharin intake by yoked rats gradually declined.

The first experiment suggested that yoked rats might possibly drink less water than conventionally trained rats. The second experiment did not confirm this effect. It is therefore concluded that the trend in the first experiment was the result of chance.

\section{GENERAL DISCUSSION}

Previous attempts to document conditioned increases in fluid acceptance have shown that carbohydrate infusions stimulate ingestion of some fluids greatly, yet have only small effects on the intake of other fluids (Ramirez, 1994b, 1996). Such large differences between stimuli made it seem possible that a nonassociative effect might be involved. Yoked controls provide a reasonably clean test for nonassociative effects, because the yoked rats receive infusions at the same times and in the same amounts as do conventionally trained rats. These groups differ only with regard to the degree to which infusions are contingent on ingestion. Nevertheless, it is important to consider potential flaws in this procedure.
According to an argument developed by Church (1964), individual variation in saccharin ingestion among rats may give rise to a difference between yoked and conventionally trained rats. However, carbohydrate-induced stimulation of intake is a robust effect that is not readily disrupted by moderate changes in training conditions. Previous studies have shown that halving the concentration of carbohydrate infused does not appreciably reduce the amount of saccharin ingested (Ramirez, 1996). Similarly, reducing the volume infused to $40 \%$ of that usually infused did not appreciably affect saccharin intake (Ramirez, 1994b). Given this degree of robustness, it does not seem likely that the subtle bias described by Church would completely obliterate any stimulation of saccharin intake in yoked rats in Experiment 1. Another possibility is that yoking per se increases or decreases intake. In Experiment 2, yoked rats showed increased saccharin ingestion earlier than did conventionally trained rats, suggesting that yoking per se might stimulate saccharin intake under certain circumstances. However, Experiment 1 indicated that, after extended training, switching yoked rats to a conventional training procedure had no effect on their intake. It therefore does not seem likely that any artifacts produced by yoking have seriously distorted the outcome.

Another concern with the procedures used in this report has to do with the ability of rats to associate flavors with long-delayed postingestive consequences (Capaldi, 1992; Capaldi et al., 1987; Elizalde \& Sclafani, 1988; E. W. Holman, 1975). This is the probable cause of the 

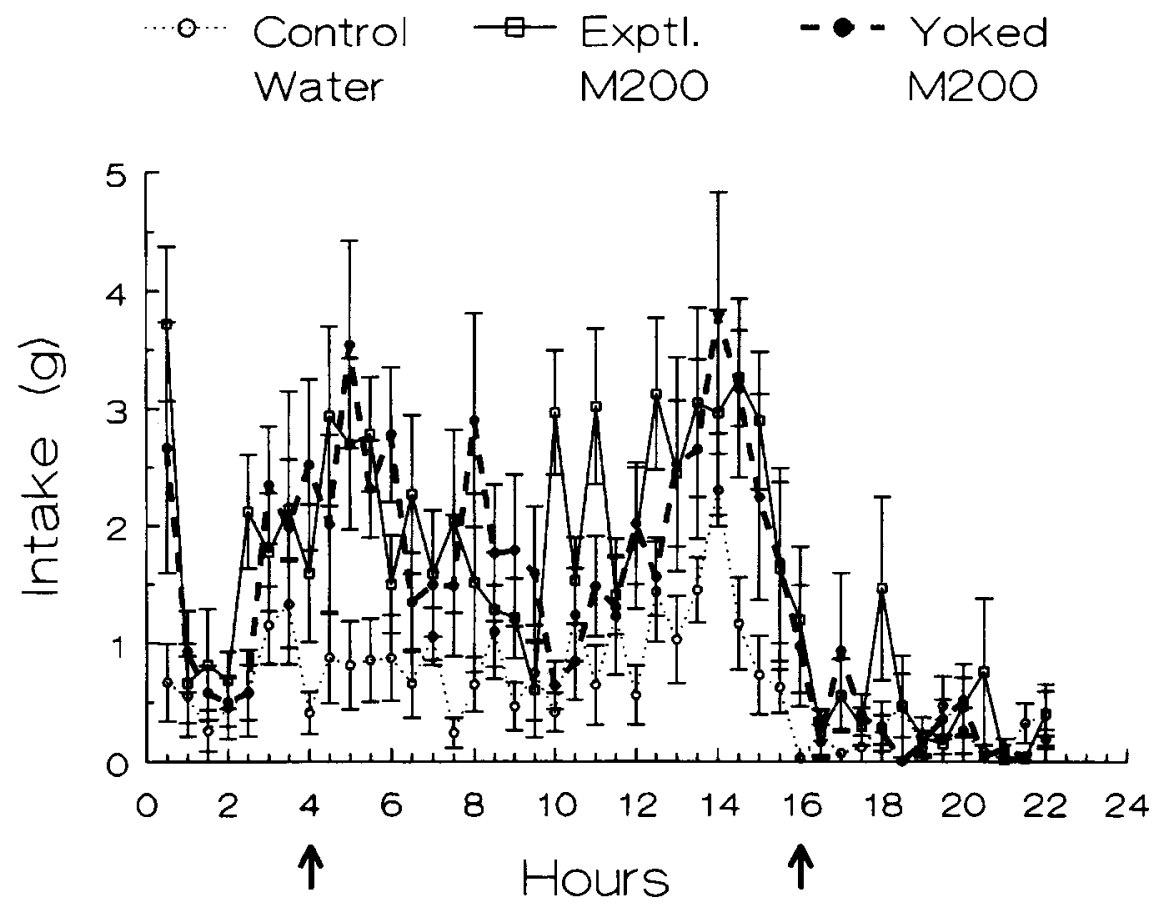

Figure 7. Oral intake $( \pm S E M)$ over successive 30-min periods during the 1st day on which saccharin was available all day long in Experiment 2. The arrows indicate when the lights went off $(4 \mathrm{~h})$ and on (16 h). Between 22 and $24 \mathrm{~h}$ the apparatus was serviced and no fluid was available.

apparent conditioning observed in the yoked rats in the first part of Experiment 2. The net result, however, is to strengthen the case for an associative mechanism. It is easy to account for the overall pattern of results in the two experiments by appealing to the effects of massed versus spaced trials on long-delay conditioning, whereas it is difficult to account for the results in terms of any known nonassociative effects.

Interactions between carbohydrate infusions and the maintenance diet could have affected the results in several different ways. Any satiating effects of the carbohydrate might reduce intake of the maintenance food and thereby reduce prandial drinking. Thus, the intake-stimulating effects of carbohydrate infusions may have been underestimated in the present report. Another possibility is that learned associations between the taste of saccharin and the postingestive effects of the maintenance food may stimulate saccharin intake (Capretta, 1964). It does not seem likely that this hypothetical factor compromised the present experiments, because there is no obvious reason why it would differentially affect intake by conventionally trained and yoked rats. A more complex concern is that the relationship between times of food ingestion and times of infusion may have differed in conventionally trained and yoked rats. If conventionally trained rats tended to ingest saccharin at some regular point in the feeding cycle (e.g., before meals), the two groups would be infused, on the average, at different states of hunger. But this factor should not have greatly distorted the results, because ad lib fed rats eat frequent meals, making it likely that the degree of hunger does not vary greatly over time.

Other tests for associative effects have been employed in previous studies; these include latent inhibition, stimulus preexposure, extinction, and stimulus generalization (Ramirez, 1994b, 1996). Since all of the evidence at hand points to an associative mechanism, it seems safe to conclude that associative conditioning is a major determinant of the intake of sweet fluids, something that has been suspected for a long time (Capretta, 1964; Le Magnen, 1954). Carbohydrate infusions rapidly doubled saccharin intake even though the amount of carbohydrate infused was modest and the animals were not deprived of food. Since responses trained with saccharin generalize to sucrose (Ramirez, 1996), this phenomenon probably reflects a mechanism that normally plays a major role in determining the rewarding properties of sugar solutions. Although it has been thought that saccharin is a less potent motivator of ingestion than sugar solutions on account of its off-taste (Morrison \& Jessup, 1977), it now appears that at least part of the difference between saccharin and sugar solutions is due to the absence of calories in saccharin.

\section{REFERENCES}

Boakes, R. A., \& Lubart, T. (1988). Enhanced preferences for a flavour following reversed flavour glucose pairing. Quarterly Journal of Experimental Psychology, 40, 49-62. 
CAPaldi, E. D. (1992). Conditioned food preferences. In D. L. Medin (Ed.), The psychology of learning and motivation (Vol. 28, pp. 1-33). San Diego: Academic Press.

Capaldi, E. D., Camprell, D. H., Sheffer, J. D., \& Bradford, J. P. (1987). Conditioned flavor preferences based on delayed caloric consequences. Journal of Experimental Psychology, 13, 150-155.

CAPRETTA, P. J. (1964). Saccharin consumption and the reinforcement issue. Journal of Comparative \& Physiological Psychology, 57, 448-450.

CHURCH, R. M. (1964). Systematic effect of random error in the yoked control design. Psychological Bulletin, 62, 122-131.

EDgington, E. S. (1987). Randomization tests (2nd ed.). New York: Marcel Dekker.

ElizALDE, G., \& Sclafani, A. (1988). Starch-based conditioned flavor preference in rats: Influence of taste, calories and CS-US Delay. Appetite, 11, 179-200.

Geiselman, P. J. (1987). Carbohydrates do not always produce satiety: An explanation of the appetite- and hunger-stimulating effects of carbohydrates. In A. N. Epstein \& A. R. Morrison (Eds.), Progress in psychobiology and physiological psychology (Vol. 12, pp. 1-46). Orlando, FL: Academic Press.

Geiselman, P. J., \& Novin, D. (1982). Sugar infusion can enhance feeding. Science, 218, 490-491.

Gibbon, J., Baldock, M. D., Locurto, C., Gold, L., \& Terrace, H. S. (1977). Trial and intertrial durations in autoshaping. Journal of Experimental Psychology: Animal Behavior Processes, 3, 264-284.

HolmaN, E. W. (1975). Immediate and delayed reinforcers for flavor preferences in rats. Learning \& Motivation, 6, 91-100.

HolmaN, G. L. (1969). Intragastric reinforcement effect. Journal of Comparative \& Physiological Psychology, 69, 432-441.

LE MAGNen, J. (1954). Le processus de discrimination par le rat blanc des stimuli sucres alimentaires et non alimentaires. Journal de Physiologie, 46, 414-418.
MANLY, B. F. J. (1991). Randomization and Monte Carlo methods in biology. London: Chapman \& Hall.

MeHIEL, R. (1991). Hedonic-shift conditioning with calories. In E. D. Capaldi \& T. L. Powley (Eds.), Taste, experience, and feeding (pp. 107 126). Washington, DC: American Psychological Association.

MorRISON, G. R., \& Jessup, A. (1977). A dual taste for saccharin in the rat. Chemical Senses \& Flavor, 2, 395-400.

RAMIREZ, I. (1994a). Glucose polymer taste is not unitary for rats. Physiology \& Behavior, 55, 355-360.

RAMIREZ, I. (1994b). Stimulation of fluid intake by carbohydrates: Interaction of taste and calories. American Journal of Physiology, 266, R682-R687.

RAMIREZ, I. (1996). Stimulus specificity in flavor acceptance learning. Physiology \& Behavior, 60, 595-610.

RAMIREZ, I. (1997). Intragastric carbohydrate exerts both intakestimulating and intake-suppressing effects. Behavioral Neuroscience, 111, 613-623.

SCLAFANI, A. (1990). Nutritionally based learned flavor preferences in rats. In E. D. Capaldi \& T. L. Powley (Eds.), Taste, experience, and feeding (pp. 139-156). Washington DC: American Psychological Association.

ToRDOFF, M. G. (1991). Metabolic basis of learned food preferences. In M. I. Friedman, M. G. Tordoff, \& M. R. Kare (Eds.), Chemical Senses: Vol. 4. Appetite and nutrition (pp. 239-260). New York: Marcel Dekker.

YouNG, W. G., \& Deutsch, J. A. (1981). The construction, surgical implantation, and use of gastric catheters and a pyloric cuff. Journal of Neuroscience Methods, 3, 377-384.

(Manuscript received January 25, 1996; revision accepted for publication January 3, 1997.) 